\title{
窄深型河湾多尺度紊流拟序结构动力稳定与 自适应特征研究
}

白玉川 ${ }^{(12}{ }^{*}$, 冀自青 ${ }^{(1)}$, 徐海玨 ${ }^{(1)}$

(1) 天津大学水利工程仿真与安全国家重点实验室, 天津 300072;

(2) 天津大学建筑工程学院河流海岸工程泥沙研究所, 天津 300072

*E-mail: ychbai@tju.edu.cn

收稿日期: 2011-11-13; 接受日期: 2012-04-19

国家自然科学基金创新研究群体科学基金(批准号: 51021004)和国家自然科学基金(批准号: 50979066, 50809045)资助项目

\begin{abstract}
摘要一定尺度范围的紊流涡体会主导河流形态的发展, 塑造出某一特定弯曲度的河段, 这 种涡体称之为“弯道造床涡体”; 弯道造床涡体与弯道相互协调关系则表现为“河流的自适应特 征”, 在以上两个概念的基础上, 本文以自然界 $\mathrm{U}$ 型河湾为背景, 从理论上分析了常曲率河湾紊 流拟序涡体结构的稳定与自适应特征. 参照流体力学相关理论, 将紊流拟序涡体结构看作一种 扰动, 计算了不同弯曲度情况下, 河湾拟序涡体的扰动增长率和扰动波数响应范围; 研究了不 同尺度紊流结构对河湾参数的响应情况, 解释了河湾得以保持的可能动力机理, 为进一步研究 河湾蠕动提供了理论基础.
\end{abstract}

关键词

弯曲河道 $\mathrm{U}$ 型河湾 造床涡体 自适应性 紊流
弯曲型河流是自然界最为常见的河流形态. 当 水流流过顺直河道时, 由于河床或河岸边界影响, 会 产生局部扰动, 进而改变原来的水流结构. 在满足一 定条件的情况下, 又会促使顺直河道发生弯曲, 形成 弯曲河流或局部河湾. 在河湾发展过程中, 某种形态 的河湾表现出相对稳定性, 具有出现机会多和维持 时间长的特点, 这种河湾也称之为最佳弯道形态 ${ }^{[1,2]}$.

近年来一些学者多从河湾动力轴线与切滩敉弯 的角度, 研究河湾水动力与河湾演变的关系 ${ }^{[3,4]}$; 随 着研究的深入, 与弯曲河流相适应的弯道紊流结构 问题, 也是近年来河流动力学中的热点课题. 国内外 不少学者通过室内试验和野外测量对河道泥沙特性、 弯道水流流速分布、水面横比降等问题进行了详细的
研究, 探讨了弯道紊流特性 ${ }^{[5 \sim 8]}$. 随着各种自由水面 跟踪方法的不断提出和紊流模型的迅速发展, 直接 利用水流数学模型对河道中局部河段进行数值模拟 已成为可能 ${ }^{[9 \sim 13]}$. 在弯道层流失稳、紊流溃灭以及床 面形态演化等理论研究方面, 近年来也取得了较大

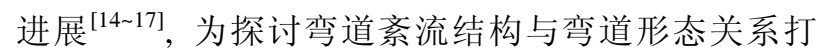
下了理论基础.

本文以自然界转弯半径较大、近似常曲率的河湾 为背景, 按照 Yalin ${ }^{[18]}$ 的观点, 将大尺度拟序涡体结 构看作是一种扰动, 采用稳定性理论分析方法 ${ }^{[19 ~ 21]}$, 研究常曲率弯曲河道中紊流结构动力多尺度演化特 征, 从理论角度寻找控制河湾形状、与河湾相适应的 “特征弯道造床涡体”. 


\section{1 理论模式}

自然界河流分为 3 种类型: 顺直、蜿蜒和分汊, 本文仅以蜿蜒型河流中常曲率河湾段为背景, 建立 水动力方程.

\section{1 常曲率弯曲河流控制方程}

1) 坐标变换关系.

以河道中心 $y=y(x)$ 为横坐标: $s$ 坐标, 以垂直于 $s$ 坐标为纵坐标: $n$ 坐标, 以垂直于河流地面的方向为 $z$ 坐标, 建立跟踪河道走向的正交曲线坐标系.

根据图 1, 曲线坐标系与直角坐标系之间的换算 关系为

$$
\begin{aligned}
& x=x_{0}+\Delta x=x_{0}-n \cos \beta=x_{0}-n \frac{\mathrm{d} y_{0}}{\mathrm{~d} s}, \\
& y=y_{0}+\Delta y=y_{0}+n \sin \beta=y_{0}+n \frac{\mathrm{d} x_{0}}{\mathrm{~d} s},
\end{aligned}
$$

其中 $\left(x_{0}, y_{0}\right)$ 与 $(x, y)$ 是直角坐标系下相距 $(\Delta x, \Delta y)$ 的河 道中的两点, $\beta$ 是点 $\left(x_{0}, y_{0}\right)$ 在新坐标系下的 $n$ 坐标与 原直角坐标系下 $x$ 坐标之间的夹角.

曲率半径:

$$
r=r(s)=\left(\frac{\mathrm{d} x_{0}}{\mathrm{~d} s} \frac{\mathrm{d}^{2} y_{0}}{\mathrm{~d} s^{2}}-\frac{\mathrm{d}^{2} x_{0}}{\mathrm{~d} s^{2}} \frac{\mathrm{d} y_{0}}{\mathrm{~d} s}\right)^{-1} .
$$

拉梅系数:

$$
\begin{aligned}
& h_{s}=\sqrt{\left(\frac{\partial x}{\partial s}\right)^{2}+\left(\frac{\partial y}{\partial s}\right)^{2}+\left(\frac{\partial z}{\partial s}\right)^{2}}=1-N, \\
& h_{n}=\sqrt{\left(\frac{\partial x}{\partial n}\right)^{2}+\left(\frac{\partial y}{\partial n}\right)^{2}+\left(\frac{\partial z}{\partial n}\right)^{2}}=1,
\end{aligned}
$$

其中 $N=n / r(s) ; C(s)=1 / r(s)$ 为弯道曲率, 当弯道接近 正余弦函数形状，则曲率等参数有如下形式:

$$
\frac{1}{r}=C \mathrm{e}^{\mathrm{i}\left(\alpha_{f} s-\omega_{g} t\right)}, N=\frac{n}{r}=C n \mathrm{e}^{\mathrm{i}\left(\alpha_{f} s-\omega_{g} t\right)},
$$

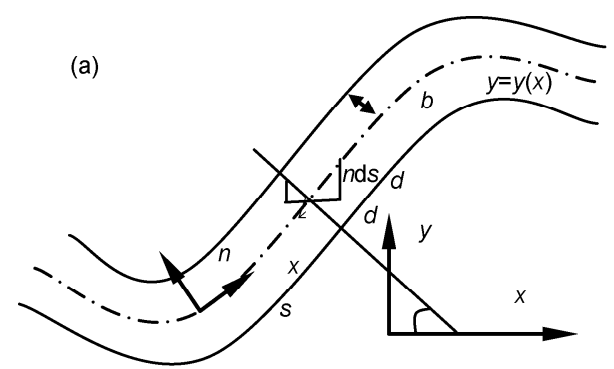

其中 $\alpha_{f}$ 为弯道的波数; $\omega_{g}$ 为弯道蠕动频率; 对于固 定弯道, $\omega_{g}=0$; 对于常曲率 $\alpha_{f}=0$.

2) 控制方程.

在新坐标系 $(s, n)$ 下, 连续性方程和水流 N-S 方 程变为

连续性方程

$$
\frac{1}{1-N} \frac{\partial u_{s}}{\partial s}-\frac{u_{n}}{(1-N) r}+\frac{\partial u_{n}}{\partial n}+\frac{\partial u_{z}}{\partial z}=0,
$$

$s$ 方向运动方程

$\frac{\partial u_{s}}{\partial t}+\frac{u_{s}}{1-N} \frac{\partial u_{s}}{\partial s}+u_{n} \frac{\partial u_{s}}{\partial n}+u_{z} \frac{\partial u_{s}}{\partial z}-\frac{u_{s} u_{n}}{(1-N) r}$

$=f_{s}-\frac{1}{\rho(1-N)} \frac{\partial P}{\partial s}+v \Delta u_{s}$

$+v\left\{\left[-\frac{2}{(1-N)} \frac{\partial u_{n}}{\partial s}-\frac{\partial u_{s}}{\partial n}-\frac{u_{s}}{(1-N) r}\right] \frac{1}{(1-N) r}\right\}$,

$n$ 方向运动方程

$\frac{\partial u_{n}}{\partial t}+\frac{u_{s}}{1-N} \frac{\partial u_{n}}{\partial s}+u_{n} \frac{\partial u_{n}}{\partial n}+u_{z} \frac{\partial u_{n}}{\partial z}-\frac{u_{s}^{2}}{(1-N) r}$

$=f_{n}-\frac{1}{\rho} \frac{\partial P}{\partial n}+v \Delta u_{m}$

$+v\left\{\left[\frac{2}{(1-N)} \frac{\partial u_{n}}{\partial s}-\frac{\partial u_{n}}{\partial n}-\frac{u_{n}}{(1-N) r}\right] \frac{1}{(1-N) r}\right\}$,

$z$ 方向运动方程

$$
\begin{aligned}
& \frac{\partial u_{z}}{\partial t}+\frac{u_{s}}{1-N} \frac{\partial u_{z}}{\partial s}+u_{n} \frac{\partial u_{z}}{\partial n}+u_{z} \frac{\partial u_{z}}{\partial z} \\
& =f_{z}-\frac{1}{\rho} \frac{\partial P}{\partial z}+v \Delta u_{z}-\frac{v}{(1-N) r} \frac{\partial u_{z}}{\partial n},
\end{aligned}
$$

其中 $u_{i}(i=s, n, z)$ 分别为 $s, n$ 和 $z$ 方向的速度; $f_{i}(i=s$, $n, z)$ 分别为 $s, n$ 和 $z$ 方向上的质量力, $f_{s}=g \sin \theta=g J$, $f_{n}=0, f_{z}=-g(1-\cos \theta) \approx-g$; 河流底坡 $J=\sin \theta ; \theta$ 为河
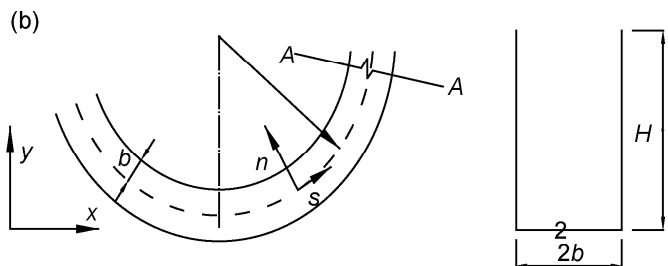

A-A

图 1 (a) 河道正交曲线坐标系; (b) 常曲率河湾或 $U$ 型河湾 
流底坡与水平面夹角; $g$ 为重力加速度; $P$ 为压力; $\Delta=\frac{1}{(1-N)^{2}} \frac{\partial^{2}}{\partial s^{2}}+\frac{\partial^{2}}{\partial n^{2}}+\frac{\partial^{2}}{\partial z^{2}} ; v$ 为液体粘滞系数和紊 动粘滞系数之和, 由于在紊流运动中, 紊动占主体, 所以取紊动粘滞系数, 具体根据 Engelund 的理论, 取 $v=0.077 U_{*} H$, 式中 $U_{*}$ 为底摩阻流速.

\section{2 无量纲化}

采用三个尺度对空间进行无量纲化, 即: 横向尺 度用半河宽 $b$, 垂向尺度用水深 $H$, 河道曲率半径用 河道最小曲率半径 $r_{m}$ (在常曲率河道中, $r_{m}$ 即为河道 中心线曲率半径, 而在变曲率河道中, 河道中心线曲 率随 $s$ 变化, 这时的 $r_{m}$ 表示曲率的最小值)等分别无 量纲化; 速度尺度用河道平均流 $U$ 无量纲化 ( $U=C \sqrt{H J}, C$ 为谢才系数, $J$ 为河流底坡); 时间尺 度用 $\frac{b}{U}$ 无量纲化.

空间量: $s=b \tilde{s}, n=b \tilde{n}, z=H \tilde{z}, \quad r=r_{m} \tilde{R}$.

速度量: $u_{s}=U \tilde{u}_{s}, u_{n}=U \tilde{u}_{n}, u_{z}=U \tilde{u}_{z}$.

压力: $p=\rho U^{2} \tilde{P}$.

时间量: $t=\frac{b}{U} \tilde{t}$.

参数: $N=\frac{n}{r}=\frac{b \tilde{n}}{r_{m} \tilde{R}}=\frac{b}{r_{m}} \tilde{N}$.

定义无量纲参数 $\psi=\frac{b}{r_{m}}, \delta=\frac{H}{b}$, 则得无量纲 连续性方程和 $\mathrm{N}-\mathrm{S}$ 方程:

$$
\frac{1}{(1-\psi \tilde{N})} \frac{\partial \tilde{u}_{s}}{\partial \tilde{s}}-\frac{\psi}{1-\psi \tilde{N}} \frac{\tilde{u}_{n}}{\tilde{R}}+\frac{\partial \tilde{u}_{n}}{\partial \tilde{n}}+\frac{1}{\delta} \frac{\partial \tilde{u}_{z}}{\partial \tilde{z}}=0,
$$

$$
\begin{aligned}
& \frac{\tilde{u}_{s}}{(1-\psi \tilde{N})} \frac{\partial \tilde{u}_{s}}{\partial \tilde{s}}+\tilde{u}_{n} \frac{\partial \tilde{u}_{s}}{\partial \tilde{n}}+\frac{1}{\delta} \tilde{u}_{z} \frac{\partial \tilde{u}_{s}}{\partial \tilde{z}}-\frac{\psi \tilde{u}_{s} \tilde{u}_{n}}{(1-\psi \tilde{N}) \tilde{R}} \\
= & f_{s} \frac{b}{U^{2}}-\frac{1}{(1-\psi \tilde{N})} \frac{\partial \tilde{p}}{\partial \tilde{s}}+\frac{1}{R e} \overline{\Delta \tilde{u}_{s}} \\
& -\frac{\psi}{\operatorname{Re}} \frac{1}{(1-\psi \tilde{N}) \tilde{R}}\left[\frac{2}{(1-\psi \tilde{N})} \frac{\partial \tilde{u}_{n}}{\partial \tilde{s}}+\frac{\partial \tilde{u}_{s}}{\partial \tilde{n}}+\frac{\psi \tilde{u}_{s}}{(1-\psi \tilde{N}) \tilde{R}}\right], \\
& \frac{\partial \tilde{u}_{n}}{\partial \tilde{t}}+\frac{\tilde{u}_{s}}{(1-\psi \tilde{N})} \frac{\partial \tilde{u}_{n}}{\partial \tilde{s}}+\tilde{u}_{n} \frac{\partial \tilde{u}_{s}}{\partial \tilde{n}}+\frac{1}{\delta} \tilde{u}_{z} \frac{\partial \tilde{u}_{n}}{\partial \tilde{z}}+\frac{\psi \tilde{u}_{s}^{2}}{(1-\psi \tilde{N}) \tilde{R}}
\end{aligned}
$$

$$
\begin{aligned}
& =f_{n} \frac{b}{U^{2}}-\frac{\partial \tilde{p}}{\partial \tilde{n}}+\frac{1}{\operatorname{Re}} \bar{\Delta}_{n} \\
& +\frac{\psi}{\operatorname{Re}} \frac{1}{(1-\psi \tilde{N})} \frac{1}{\tilde{R}}\left[\frac{2}{(1-\psi \tilde{N})} \frac{\partial \tilde{u}_{s}}{\partial \tilde{s}}-\frac{\psi \tilde{u}_{n}}{(1-\psi \tilde{N}) \tilde{R}}-\frac{\partial \tilde{u}_{n}}{\partial \tilde{n}}\right] \\
& \quad \frac{\partial \tilde{u}_{z}}{\partial \tilde{t}}+\frac{\tilde{u}_{s}}{(1-\psi \tilde{N})} \frac{\partial \tilde{u}_{z}}{\partial \tilde{s}}+\tilde{u}_{n} \frac{\partial \tilde{u}_{z}}{\partial \tilde{n}}+\frac{1}{\delta} \tilde{u}_{z} \frac{\partial \tilde{u}_{z}}{\partial \tilde{z}} \\
& \quad=\frac{f_{z} b}{U^{2}}-\frac{1}{\delta} \frac{\partial \tilde{p}}{\partial \tilde{z}}+\frac{1}{\operatorname{Re}} \bar{\Delta}_{\bar{u}}-\frac{\psi}{\operatorname{Re}} \frac{1}{(1-\psi \tilde{N}) \tilde{R}} \frac{\partial \tilde{u}_{z}}{\partial \tilde{n}},
\end{aligned}
$$

其中 $\bar{\Delta}=\frac{1}{(1-\psi \tilde{N})^{2}} \frac{\partial^{2}}{\partial \tilde{s}^{2}}+\frac{\partial^{2}}{\partial \tilde{n}^{2}}+\frac{1}{\delta^{2}} \frac{\partial^{2}}{\partial \tilde{z}^{2}}$, 雷诺数 $R e=$ $U b / v=12.98 \frac{U}{U_{*}} \frac{1}{\delta}=25.98 \sqrt{\frac{2}{\lambda}} \frac{1}{\delta}$.

\section{3 摄动分析}

按照流体力学中处理紊流拟序结构的理论方 法 ${ }^{[20 ~ 24]}$, 对方程组(5) (8)进行摄动求解, 取

$$
\left[\begin{array}{c}
\tilde{u}_{s} \\
\tilde{u}_{n} \\
\tilde{u}_{z} \\
\tilde{p}
\end{array}\right]=\sum_{i=0}^{\infty}\left[\begin{array}{c}
u_{s \psi i} \\
u_{n \psi i} \\
u_{z \psi i} \\
p_{\psi i}
\end{array}\right] \psi^{i}+\varepsilon_{T}\left[\begin{array}{c}
\tilde{u}_{s T} \\
\tilde{u}_{n T} \\
\tilde{u}_{z T} \\
\tilde{p}_{T}
\end{array}\right] .
$$

一般情况下, 河流的半河宽远较河流曲率半径 为小, 弯曲度 $\psi$ 为一小参数, 与其相应的项为弯曲形 状引起的修正量, 是对顺直河道修正, 代表弯道二 次、三次及高次流的部分; $\varepsilon_{T}$ 为拟序参数, 其对应的 量代表拟序扰动量. 考虑 $\psi \tilde{N}$ 为小量, 则有

$$
\begin{aligned}
& \frac{1}{1-\psi \tilde{N}} \approx 1+\psi \tilde{N}+\cdots, \\
& \frac{1}{(1-\psi \tilde{N})^{2}} \approx 1+2 \psi \tilde{N}+\cdots,
\end{aligned}
$$

则方程(5) (8)可转变为 $\psi^{i}$ 和 $\varepsilon_{T}$ 的一系列方程.

\subsection{1 基本量与弯曲修正量分析}

将(9)式中等号右边第一项中 “基本量(顺直部分 $\left.\psi^{i}, i=0\right)+$ 弯曲扰动部分 $\left(\psi^{i}, i=1\right)$ ” 代入方程(5) (8), 弯 曲的影响可仅考虑到 $\psi$ 一阶量, 其余略去.

1) $\psi^{0}$ 项求解.

与 $\psi^{0}$ 相关的项代表基本量 (顺直部分), 如: 流 动量 $\tilde{u}_{s \psi 0}, \tilde{u}_{n \psi 0}, \tilde{u}_{z \psi 0}$ 和压力 $\tilde{p}_{\psi 0}$; 对于顺直河道, 又有: 
$\tilde{u}_{n \psi 0}=0, \partial \tilde{u}_{z \psi 0} / \partial \tilde{z}=\partial \tilde{u}_{s \psi 0} / \partial \tilde{s}=0, \tilde{u}_{z \psi 0}=0$ 和 $\partial / \partial t=0$. 因此, 流动基本量满足的方程变为

$$
\begin{gathered}
\frac{\partial \tilde{u}_{s \psi 0}}{\partial \tilde{s}}=0, \\
g J \frac{b}{U^{2}}-\frac{\partial \tilde{p}_{\psi 0}}{\partial \tilde{s}}+\frac{1}{R e} \bar{\Delta} \tilde{u}_{s \psi 0}=0, \\
-\frac{\partial \tilde{p}_{\psi 0}}{\partial \tilde{n}}=0, \\
-\frac{g H}{U^{2}}-\frac{\partial \tilde{p}_{\psi 0}}{\partial \tilde{z}}=0 .
\end{gathered}
$$

考虑水面条件：当 $\tilde{z}=\tilde{\eta}, \tilde{p}_{\psi 0}=\tilde{p}_{a}$ ， 所以

$$
\left[\frac{\partial^{2}}{\partial \tilde{n}^{2}}+\left(\frac{1}{\delta}\right)^{2} \frac{\partial^{2}}{\partial \tilde{z}^{2}}\right] \tilde{u}_{s \psi 0}=\frac{R e}{F_{r}^{2}}\left[\frac{\partial \tilde{\eta}}{\partial \tilde{s}}-\frac{J}{\delta}\right] .
$$

河岸边界条件: $n= \pm 1 ; u_{s y 0}( \pm 1, z)=0$.

水面条件: $z=\tilde{\eta},\left.\frac{\partial \tilde{u}_{s 0}}{\partial \tilde{z}}\right|_{\tilde{\eta}}=0$.

河底条件: $z=-\tilde{h}, u_{s 0}(-\tilde{h})=0$.

在窄深情况下, $\delta=\frac{H}{b}>>1, \frac{1}{\delta}<<1, \frac{1}{\delta^{2}}<<1$, 转化为二维问题, 则方程(12)简化为

$$
\frac{\partial^{2} \tilde{u}_{s y 0}}{\partial \tilde{n}^{2}}=\frac{R e}{F_{r}^{2}}\left[\frac{\partial \tilde{\eta}}{\partial \tilde{s}}-\frac{J}{\delta}\right] .
$$

求解(13)式, 并结合河岸边界条件: $\tilde{n}= \pm 1, u_{s}=$ 0 , 得

$$
\tilde{u}_{s \psi 0}=\frac{1}{2} \frac{R e}{F_{r}^{2}}\left[\frac{\partial \tilde{\eta}}{\partial \tilde{s}}-\frac{J}{\delta}\right]\left(\tilde{n}^{2}-1\right) .
$$

2) $\psi^{1}$ 项, 即弯曲修正量.

与 $\psi^{1}$ 相应的量 $\tilde{u}_{s \psi 1}, \tilde{u}_{n \psi 1}, \tilde{u}_{z \psi 1}$ 和 $\tilde{p}_{\psi 1}$ 代表河流 弯曲所诱发的修正量. 在窄深情况下, $1 / \delta=b / H \ll 1$, 连续性方程、动量方程变为

$$
\begin{gathered}
\frac{\partial \tilde{u}_{s \psi 1}}{\partial \tilde{s}}+\frac{\partial \tilde{u}_{n \psi 1}}{\partial \tilde{n}}=0 \\
\frac{\partial \tilde{u}_{s \psi 1}}{\partial t}+\tilde{u}_{s \psi 0} \frac{\partial \tilde{u}_{s \psi 1}}{\partial \tilde{s}}+\tilde{u}_{n \psi 1} \frac{\partial \tilde{u}_{\psi s 0}}{\partial \tilde{n}} \\
=-\frac{\partial \tilde{p}_{1}}{\partial \tilde{s}}-\tilde{N} \frac{\partial \tilde{p}_{\psi 0}}{\partial \tilde{s}}+\frac{1}{R e} \bar{\Delta} \tilde{u}_{s \psi 1}-\frac{1}{R e} \frac{1}{\tilde{R}} \frac{\partial \tilde{u}_{s \psi 0}}{\partial \tilde{n}}, \\
\frac{\partial \tilde{u}_{n \psi 1}}{\partial t}+\tilde{u}_{s \psi 0} \frac{\partial \tilde{u}_{n \psi 1}}{\partial \tilde{s}}+\frac{\tilde{u}_{s \psi 0}^{2}}{\tilde{R}}=-\frac{\partial \tilde{p}_{\psi 1}}{\partial \tilde{n}}+\frac{1}{R e} \bar{\Delta}_{n \psi 1},
\end{gathered}
$$

$$
\frac{\partial \tilde{u}_{z \psi 1}}{\partial t}+\tilde{u}_{s \psi 0} \frac{\partial \tilde{u}_{z \psi 1}}{\partial \tilde{s}}=\frac{1}{R e} \bar{\Delta} \tilde{u}_{z \psi 1} .
$$

结合边界条件可知 $\tilde{u}_{z \psi 1}=0$. 因此, 速度 $\tilde{u}_{s \psi 1}$, $\tilde{u}_{n \psi 1}$ 和压力 $\tilde{p}_{\psi 1}$ 将变为二维情况.

当蜿蜒河道为常曲率的情况, 则无量纲的弯曲 参数变为如下形式:

$$
\frac{1}{\tilde{R}}=\hat{\tilde{C}} ; \quad \tilde{N}=\frac{\tilde{n}}{\tilde{R}}=\tilde{C} \tilde{n}=\hat{\tilde{C}} \tilde{n} .
$$

在窄深型常曲率蜿蜒河流情况下，与 $\psi^{1}{ }^{1}$ 相应的 修正量其解可设为

$$
\left[\begin{array}{c}
\tilde{u}_{s \psi 1} \\
\tilde{u}_{n \psi 1} \\
\tilde{p}_{\psi 1}
\end{array}\right]=\left[\begin{array}{c}
\hat{\tilde{u}}_{s \psi 1}(\tilde{n}) \\
\hat{\tilde{u}}_{n \psi 1}(\tilde{n}) \\
\hat{\tilde{p}}_{\psi 1}(\tilde{n})
\end{array}\right] .
$$

结合(15)和(16)式可得

$$
D \hat{\tilde{u}}_{n \psi 1}=0,
$$

$$
\begin{aligned}
D \tilde{u}_{s \psi 0} \hat{\tilde{u}}_{n \psi 1}= & -\hat{\tilde{N}} \frac{\partial \tilde{p}_{\psi 0}}{\partial s}+\frac{1}{R e} D^{2} \hat{\tilde{u}}_{s \psi 1}-\frac{1}{R e} \frac{\partial \tilde{u}_{s \psi 0}}{\partial \tilde{n}}\left(\frac{1}{\hat{\tilde{R}}}\right), \\
& \frac{1}{\hat{\tilde{R}}} \tilde{u}_{s \psi 0}^{2}=-D \hat{\tilde{\tilde{p}}}_{\psi 1}+\frac{1}{\operatorname{Re}} D^{2} \hat{\tilde{u}}_{n \psi 1} .
\end{aligned}
$$

由(18)和(19)式及边界条件 $\hat{\tilde{u}}_{n \psi 1}( \pm 1)=0$ 得

$$
\hat{\tilde{u}}_{s \mu 1}=-\frac{1}{6} \frac{\operatorname{Re}}{F_{r}^{2}}\left(\frac{2 \partial \tilde{\eta}}{\partial \tilde{s}}-\frac{J}{\delta}\right) \hat{\tilde{C}}\left(n-\tilde{n}^{3}\right) .
$$

\subsection{2 拟序扰动量稳定特征}

与顺直河道相比, 由于河道的弯曲特性, 改变了 原来流动失稳模式, 河道内的基本流被重新修正, 公 式(9)在小弯曲或微弯曲情况下, $\psi$ 的高价量可以略 去; 同时考虑窄深情况，根据连续方程、动量方程和 边界条件, 得 $\tilde{u}_{z T} \approx 0$, 拟序扰动方程变为二维情况. 在常曲率窄深情况下, 基本流写为

$$
\left[\begin{array}{l}
\tilde{U}_{s} \\
\tilde{U}_{n} \\
\tilde{P}
\end{array}\right]=\left[\begin{array}{l}
\tilde{u}_{s \psi 0} \\
\tilde{u}_{n \psi 0} \\
\tilde{p}_{\psi 0}
\end{array}\right]+\psi\left[\begin{array}{l}
\hat{\tilde{u}}_{s \psi 1}(\tilde{n}) \\
0 \\
\hat{\tilde{p}}_{\psi 1}(\tilde{n})
\end{array}\right] .
$$

扰动量解的形式写为

$$
\left[\begin{array}{c}
\tilde{u}_{s T} \\
\tilde{u}_{n T} \\
\tilde{p}_{T}
\end{array}\right]=\left[\begin{array}{c}
\hat{\tilde{u}}_{s T}(\tilde{n}) \\
\hat{\tilde{u}}_{n T}(\tilde{n}) \\
\hat{\tilde{p}}_{T}(\tilde{n})
\end{array}\right] \mathrm{e}^{\mathrm{i}\left[\alpha_{T} \tilde{s}-\omega_{T} \tilde{i}\right]} .
$$


记 $D=\mathrm{d} / \mathrm{d} \tilde{n}, D^{2}=\mathrm{d}^{2} / \mathrm{d} \tilde{n}^{2}$ 得到拟序扰动的幅值方 程:

$$
\begin{aligned}
& \frac{\mathrm{i} \alpha_{T}}{(1-\psi \tilde{N})} \hat{\tilde{u}}_{s T}=\left[\frac{\psi}{(1-\psi \tilde{N})}-D\right] \hat{\tilde{u}}_{n T}, \\
& \frac{1}{\operatorname{Re}}\left[D^{2}-\frac{\alpha_{T}^{2}}{(1-\psi \tilde{N})^{2}}\right] \hat{\tilde{u}}_{s T}-\frac{\psi}{\operatorname{Re}} \frac{1}{(1-\psi \tilde{N})} \frac{1}{\tilde{R}} D \hat{\tilde{u}}_{s T} \\
& -\frac{\psi}{\operatorname{Re}} \frac{\psi}{(1-\psi \tilde{N})^{2}} \frac{\hat{\tilde{u}}_{s T}}{\tilde{R}^{2}}+\mathrm{i} \omega_{T} \hat{\tilde{u}}_{s T}-\frac{\mathrm{i} \alpha_{T} \tilde{U}_{s}}{(1-\psi \tilde{N})} \hat{\tilde{u}}_{s T} \\
& =\frac{\mathrm{i} \alpha_{T}}{(1-\psi \tilde{N})} \hat{\tilde{\tilde{P}}}_{T}+D \tilde{U}_{s} \hat{\tilde{u}}_{n T}-\frac{\psi}{(1-\psi \tilde{N})} \frac{\tilde{U}_{s}}{\tilde{R}} \hat{\tilde{u}}_{n T} \\
& +\frac{\psi}{\operatorname{Re}} \frac{2 \mathrm{i} \alpha_{T}}{(1-\psi \tilde{N})^{2}} \frac{1}{\tilde{R}} \hat{\tilde{u}}_{n T}, \\
& \frac{1}{\operatorname{Re}}\left[\frac{-\alpha_{T}^{2}}{(1-\psi \tilde{N})^{2}}+D^{2}\right] \hat{\tilde{u}}_{n T}+\mathrm{i} \omega_{T} \hat{\tilde{u}}_{n T}-\frac{\mathrm{i} \alpha_{T} \tilde{U}_{s}}{(1-\psi \tilde{N})} \hat{\tilde{u}}_{n T} \\
& +\frac{\psi}{\operatorname{Re}}\left\{\frac{1}{(1-\psi \tilde{N})} \frac{1}{\tilde{R}}\left[-\frac{\psi}{(1-\psi \tilde{N})} \frac{\hat{\tilde{u}}_{n T}}{\tilde{R}}-D \hat{\tilde{u}}_{n T}\right]\right\} \\
& =D \hat{\tilde{P}}_{T}+\frac{\psi}{1-\psi \tilde{N}} \frac{2 \tilde{U}_{s}}{\tilde{R}} \hat{\tilde{u}}_{s T}-\frac{\psi}{R e} \frac{1}{\tilde{R}} \frac{2 \mathrm{i} \alpha_{T} \hat{\tilde{u}}_{S T}}{(1-\psi \tilde{N})^{2}} .
\end{aligned}
$$

解(22) (24)式, 在小弯曲或微弯曲情况下, 略去 $\psi$ 的高阶量, 得以下形式:

$$
F_{0}\left(\psi^{(0)}\right)+F_{1}\left(\psi^{(1)}\right)=0,
$$

其中 $\psi$ 的零阶量和一阶量分别展开为

$$
\begin{gathered}
F_{0}\left(\psi^{(0)}\right)=\left[D^{4} \hat{\tilde{u}}_{n T}+\frac{\alpha_{T}^{4}}{(1-\psi \tilde{N})^{4}} \hat{\tilde{u}}_{n T}-\frac{2 \alpha_{T}^{2}}{(1-\psi \tilde{N})^{2}} D^{2} \hat{\tilde{u}}_{n T}\right] \\
-\mathrm{i} \operatorname{Re}\left[\frac{\alpha_{T}}{(1-\psi \tilde{N})} \tilde{U}_{s}-\omega_{T}\right]\left[D^{2} \hat{\tilde{u}}_{n T}-\frac{\alpha_{T}^{2}}{(1-\psi \tilde{N})^{2}} \hat{\tilde{u}}_{n T}\right] \\
+\mathrm{i} \operatorname{Re} \frac{\alpha_{T}}{(1-\psi \tilde{N})} D^{2} \tilde{U}_{s} \hat{\tilde{u}}_{n T}, \\
F_{1}\left(\psi^{(1)}\right)=\frac{\psi}{(1-\psi \tilde{N})}\left\{-D^{3} \hat{\tilde{u}}_{n T}-\frac{5}{\tilde{R}} D^{3} \hat{\tilde{u}}_{n T}\right. \\
+\frac{1}{R} \frac{\alpha_{T}^{2}}{(1-\psi \tilde{N})^{2}} D \hat{\tilde{u}}_{n T}+\frac{\alpha_{T}^{2}}{(1-\psi \tilde{N})^{2}} D \hat{\tilde{u}}_{n T} \\
+\mathrm{i} \alpha_{T} \operatorname{Re} \frac{1}{R} \frac{2-\psi \tilde{N}}{1-\psi \tilde{N}} \tilde{U}_{s} D \hat{\tilde{u}}_{n T}+\mathrm{i} \alpha_{T} R e
\end{gathered}
$$

$$
\begin{aligned}
& \times \frac{\tilde{U}_{s}}{(1-\psi \tilde{N})} D \hat{\tilde{u}}_{n T}-\mathrm{i} \omega_{T} \operatorname{ReD} \hat{\tilde{u}}_{n T} \\
& -2 \mathrm{i} \omega_{T} \operatorname{Re} \frac{1}{\tilde{R}} D \hat{\tilde{u}}_{n T}-\mathrm{i} \alpha_{T} \operatorname{Re} \frac{2-\psi \tilde{N}}{(1-\psi \tilde{N})} \frac{1}{R} \\
& \left.\times D \tilde{U}_{s} \hat{\tilde{u}}_{n T}+\mathrm{i} \alpha_{T} \operatorname{Re} \frac{1}{(1-\psi \tilde{N})} D \tilde{U}_{s} \hat{\tilde{u}}_{n T}\right\} .
\end{aligned}
$$

在时间模式不稳定问题中, $\alpha_{T}$ 是扰动波的波数, 为实数. $\omega_{T}=\alpha_{T} c$ ( $c$ 代表波速) 是扰动波的频率, 为 复数, 其虚部代表由 $\left(\alpha_{T}, c\right)$ 表达的扰动量的增长率 或衰减率. 方程(25)的特征值满足的关系式可表达为

$$
F\left(\tilde{\alpha}_{T}, R e, \tilde{c}_{T}, \tilde{R}, \psi, \frac{J}{\delta}\right)=0 .
$$

方程(28)就代表了描述河湾拟序结构稳定特征 的 Orr-Sommerfeld 方程. 从该方程可以看出, 不同尺 度紊流结构能否长时间存在, 除与自身特征参数 $\left(\tilde{\alpha}_{T}, \tilde{c}_{T}\right)$ 有关外, 主要取决于流动雷诺数 $R e$ 、河湾转 弯半径 $\tilde{R}$ 、弯曲参数 $\psi$ 、坡度 $J$ 及深宽比 $\delta$ 等参数.

\section{2 河湾多尺度紊流特性计算结果及分析}

通过计算发现，在相同的水力条件下，河道紊流 中可能出现增长率不同的多个扰动量, 这些扰动量 呈现不同的频率和波数特征, 将其分为一系列的特 征模态, 定义增长率最大的模态为最不稳定模态.

\section{1 多尺度紊流模态各波段稳定特征}

图 2 显示了顺直河道 (弯曲参数 $\psi=0$ ) 和弯曲河道 (河湾, 弯曲参数 $\psi=0.1$ )中雷诺数 $R e=10000,30000$, 60000 的最不稳定模态的特征谱.

河湾中紊流存在各种不同尺度的拟序扰动波, 各波段呈现不同的发展趋势. 在 $\alpha=1$ 的附近有一段 $\omega_{i}>0$ 的不稳定波段, 这一波段中的拟序扰动波将会 逐渐增长, 而该波段左右的长波段和短波段的扰动 波则会逐渐衰减. 短波段部分衰减很快, 并随着波数 的增大逐渐趋于一稳定的衰减率; 长波段内扰动衰 减相对缓慢, 并有一极小值, $\alpha$ 趋于 0 的超长波段增 长率为 0 , 可视为恒定平均流.

随着雷诺数的增大, 不稳定波段向长数段移动, 河道中更易于存在较大波长的波动; 随着雷诺数的 增大, 长波段扰动和短波段中的小尺度扰动衰减均 减缓, 波谱更加平坦, 更易于各尺度涡的保持. 

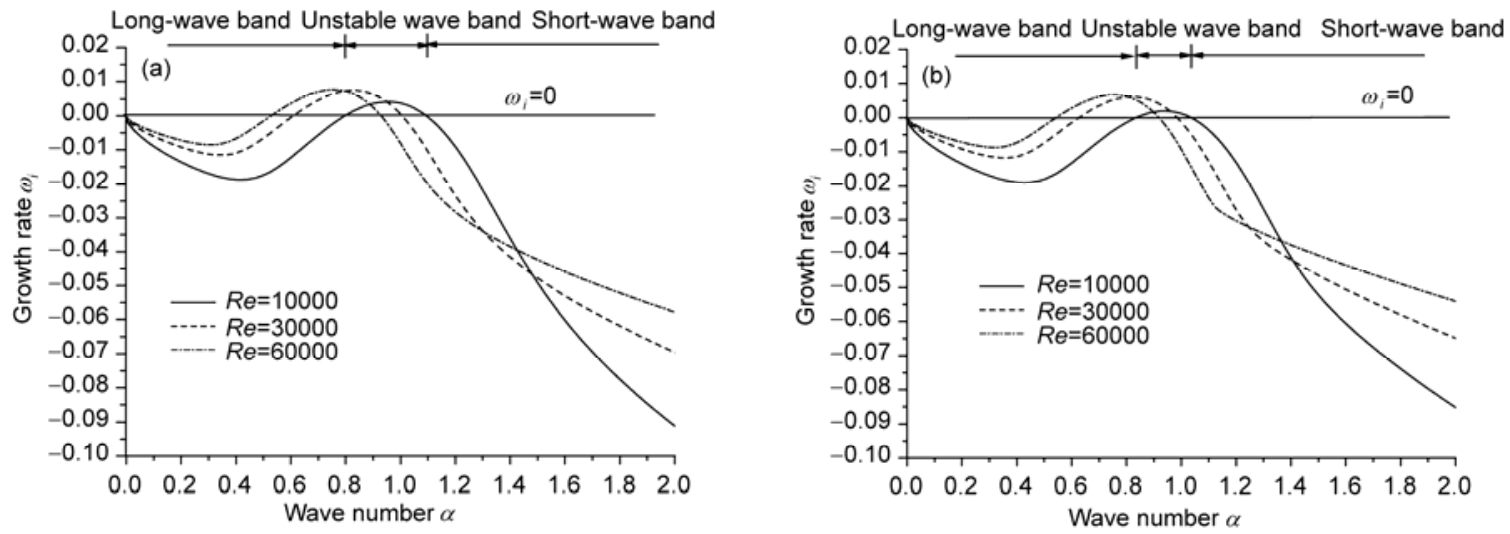

图 2 不同水动力强度下的河道特征波谱

(a) 顺直河道(弯曲度 $\psi=0$ ); (b) 弯曲河道(弯曲度 $\psi=0.1$ )

图 3 为顺直河道与弯曲河道段(河湾)的特征谱对 比. 可以看出, 河湾对河道中的特征波有一定的选择 性, 与顺直河道相比, 弯曲河段内不稳定波段缩窄; 长波段扰动特征频率及其衰减速率略微增大但变化 不大; 短波段中的小尺度扰动衰减减缓, 特征频率增 大. 河道弯曲程度对于不稳定波段和短波段扰动影 响较为明显.

\section{2 多尺度紊流特征谱模态交叉、合并与分离现象}

河湾中紊流有许多特征模态，这些模态各有其 不同的演化增减特征，增减特性不同其占据的地位 和作用不同. 特征谱模态排列在不同尺度会有所变 化, 在某一尺度上的最不稳定模态在另一尺度上可 能不再是最不稳定的模态, 如图 4 和 5 所示.

图 4 为顺直河道中雷诺数 $R e=30000$ 时的紊流模

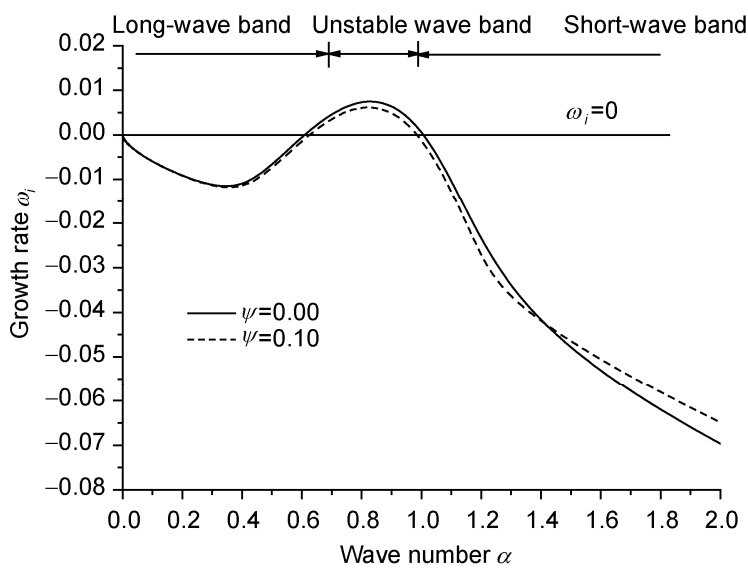

图 3 顺直河道与弯曲河段(河湾)紊流特征谱对比 $(\operatorname{Re}=\mathbf{3 0 0 0 0})$

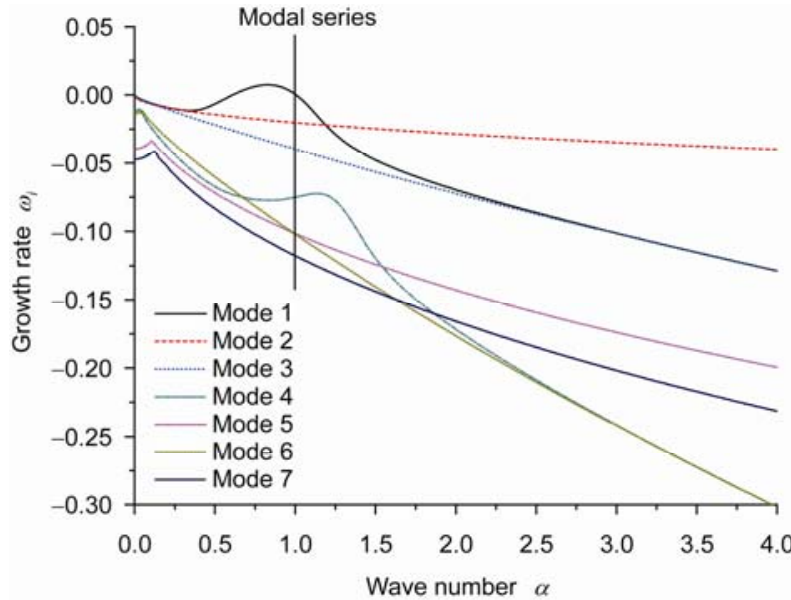

图 4 顺直河道紊流模态交叉与合并

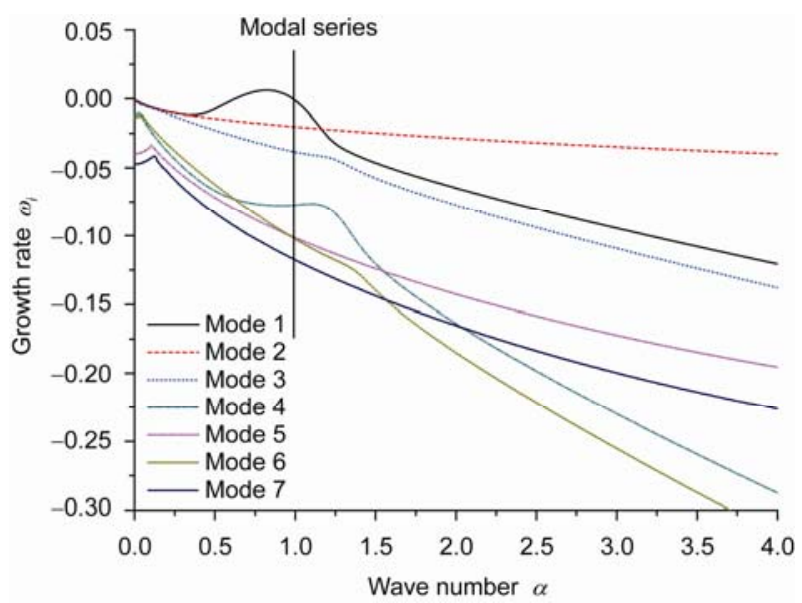

图 5 河湾中的紊流特征模态交叉与分离 
态特征谱. 为便于分析, 将波数 $\alpha=1$ 时的前 7 个模态按 扰动增长速率由大到小排列, 按顺序记为模态 1-7. 从 图 4 中可以看出, 各模态的特征谱有交叉、合并的现象.

在短波段内, 模态 1 和 2 有交叉, 并逐渐与模态 3 融合, 模态 4 与模态 5 和 6 有交叉, 并与模态 7 融 合; 在长波段内, 模态 1-3、模态 4-5 也分别融合.

不稳定波段与短波段内各有不同的模态主导. 不稳定波段内, 模态 1 最不稳定模态, 占据主导地位; 在短波段内, 随时间增长, 模态 2 虽然衰减, 但模态 1 衰减比模态 2 快得多, 模态 2 占仍主导地位.

由于模态 2 对于各小(波长)尺度的扰动来说衰减速 率相差不大, 因此, 顺直河道内存在各种尺度的小浴.

图 5 为河湾(弯曲系数 $\psi=0.1$ )中雷诺数 $R e=30000$ 的紊流模态特征谱. 河湾中也存在各模态特征谱的 交叉现象, 但是并存在不顺直河道中的模态融合现 象, 转而出现模态的分离现象 (如模态 1 与模态 3 、模 态 6 与模态 7). 其原因是弯曲河道中不同模态的扰动 增长率随弯曲程度变化而变化, 如图 6 所示模态 1 和 模态 3 的增减趋势正好相反, 使得在顺直河道中同速 率衰减的不同模态在弯曲河道中不再同速率衰减, 因而发生了图 5 所示的模态分离现象.

\section{3 多尺度紊流模态特征波速及其增长率变化特征}

将不同弯曲程度河道中的各种模态按其稳定特

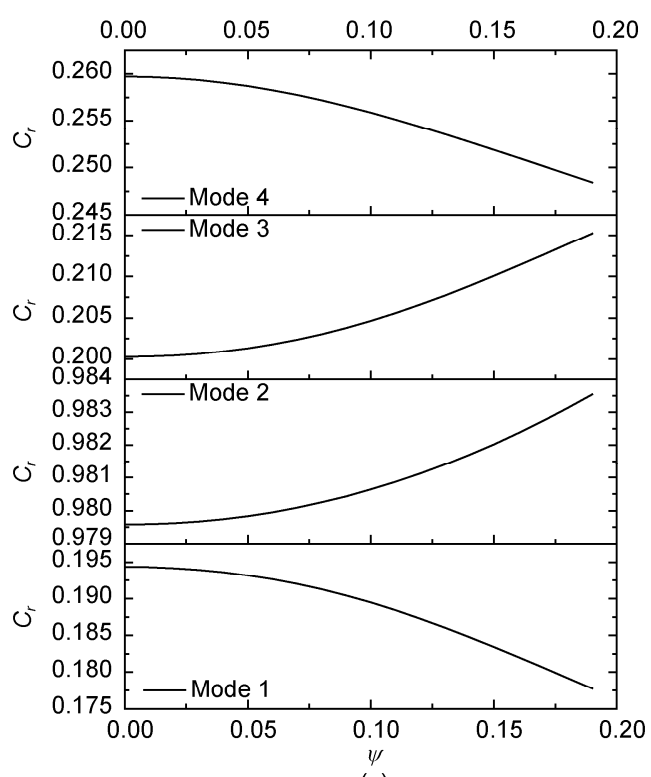

(a)
性(模态按增长率由高到低依次排列), 取最稳定的几 个模态, 对其特征进行分析.

本文计算了雷诺数 $R e=30000$ 时, 波数 $\alpha=1$ 的扰 动波随弯曲程度不同扰动波波速和波速增长率的变 化, 按增长率由大到小顺序排列各模态, 依次记为模 态 1-4, 计算结果如图 6 所示.

由图 6 可见, 河道弯曲程度对紊流中各模态的影 响程度不尽相同. 随弯曲度增大, 最不稳定的模态 (模态 1)的特征波速逐渐减小, 其增长率也逐渐减小, 出现由正转负, 这种趋势使得弯曲河道的流态比顺 直河道的流态更为稳定.

\section{3 河湾紊流拟序特征量的形态分布}

\section{1 弯曲度对紊流结构横向分布的影响}

弯曲河岸边界本身即是一种大尺度的几何扰动, 不仅改变了基本流的分布形态，同时还会对紊流拟 序结构量(扰动流、浴量、切应力)产生大的影响.

图 7 为弯曲河道中的基本流流速剖面 $(\psi=0.1)$. 可以看出由于弯曲的出现, 二次流出现反对称分布, 导致整体流速分布呈不对称分布，主流线偏向凹岸 一侧，使得凹岸流速梯度和切应力均大于凸岸，这也 正是窄深型微弯河湾逐步向有限弯曲河湾演变的动 力机理之一.

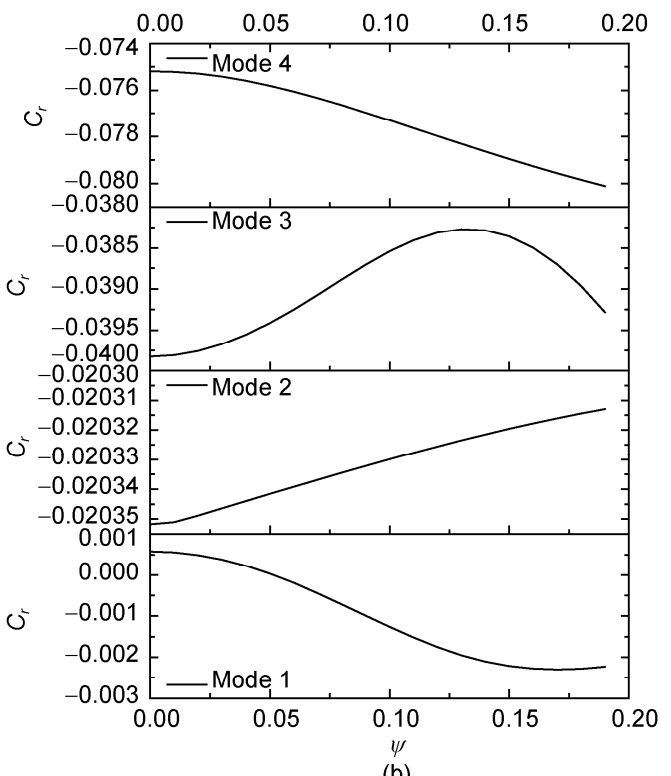

(b)

图 6 紊流中的不同模态特征

(a) 特征波速; (b) 波速增长率 


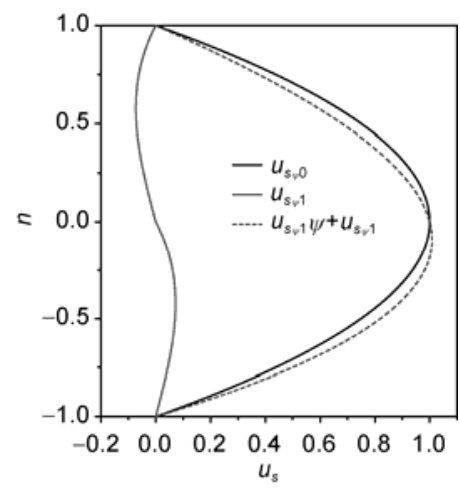

图 7 弯道基本流与二次流流速剖面 $(\psi=0.1)$

针对不同弯曲系数的河湾, 在雷诺数 $R e=30000$, 波数 $\alpha=1$ 情况下, 计算了紊流拟序扰动的流速、浴 量、扰动流速相关量的形态特征, 见图 8 10.

图 8 为不同弯曲程度下的扰动流速剖面图. 可以 看出: 顺直河道的纵向扰动流速关于河道呈反对称 分布, 而横向扰动流速和浴量呈对称分布. 在弯道中, 由于基本流向凹岸偏斜, 凹岸的纵向扰动流速( $s$ 方向) 和浴量均大于凸岸; 扰动流速剖面和涡量剖面也向 凹岸偏斜; 而横向扰动流速 $(n$ 方向)较顺直河道为小, 且基本对称略偏向凸岸.

图 9 为不同弯曲程度下的扰动流涡量剖面图. 在 近壁面边界层区域, 扰动涡量变化较大, 并有两个极 值, 一个为扰动浴量最大值, 另一个为反向浴量极值; 河道中心区域涡量很小; 弯曲度对凹岸近壁涡和反 向浴影响最为明显, 而在凸岸近壁处和中心区域则 影响较弱.

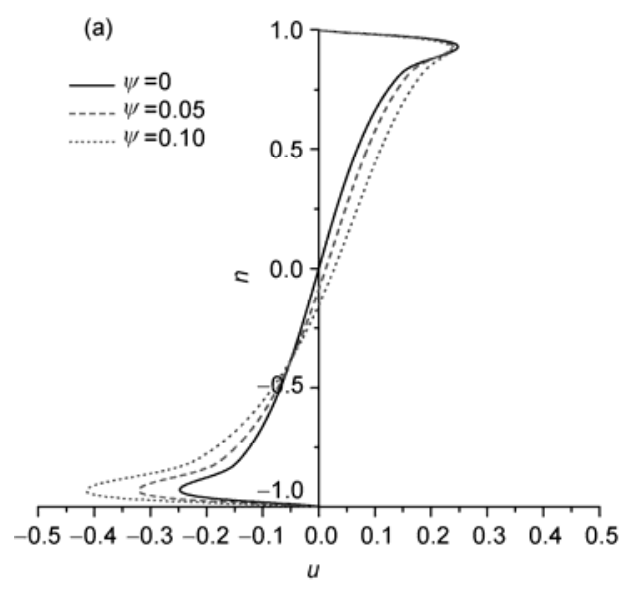

图 8 不同弯曲程度河流中扰动流速剖面

(a) 扰动流速 $u$ ( $s$ 方向); (b) 扰动流速 $v$ ( $n$ 方向)

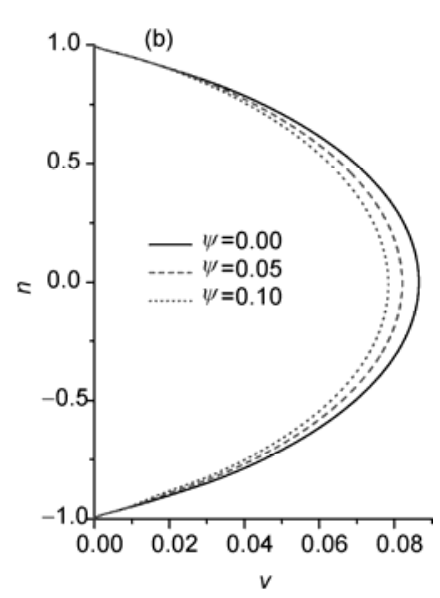

图 10 为不同弯曲程度河湾中扰动流速相关量剖 面图, 它相当于雷诺应力或扰动切应力. 可以看出, 顺直河道扰动切应力关于河道中轴线反对称分布, 凹岸雷诺应力为正, 凸岸为负; 随着弯曲程度增大, 扰动切应力向凹岸偏移, 并且切应力值也随之显著 增大, 这意味着, 相同水流强度下, 弯曲河道比顺直 河道更易发生岸壁冲蚀, 扰动切应力的不对称分布 使得弯道中凹岸冲蚀、凸岸淤积, 这是微弯河流逐步 向有限弯曲演变的另一动力因素.

\section{2 顺直河道与弯曲河道的紊动拟序流场}

图 11 为紊流拟序扰动(雷诺数 $R e=30000$, 波数 $\alpha=1$ )的流场平面分布. 可以看出, 在顺直河道中, 紊 流拟序结构为绕河流中心线上一点旋转的系列漩浴, 并向下游传播; 在弯曲河段(河湾)中, 紊流拟序结构 同样也是这样一种漩浴, 但其中心偏向凹岸. 图中不 同的颜色表示浴量大小, 河道中扰动浴量在近壁出 最大, 两壁浴量沿纵向交替出现.

\section{3 河湾紊流多模态的结构特征}

取不同弯曲程度河湾中最稳定的几个模态, 对 其结构特征进行分析, 不同的模态在扰动流速剖面 上有不同的剖面形状.

本文计算了弯曲度 $\psi=0.1$, 雷诺数 $R e=30000$, 波 数 $\alpha=1$ 时的前 3 个模态的扰动流速剖面和扰动切应 力在 $n$ 方向的变化, 并归一化处理, 见图 12 .

由图 12 可以看出, 弯曲河道(河湾)中, 各模态扰 动流速和切应力剖面均表现出向凹岸偏移的趋势, 


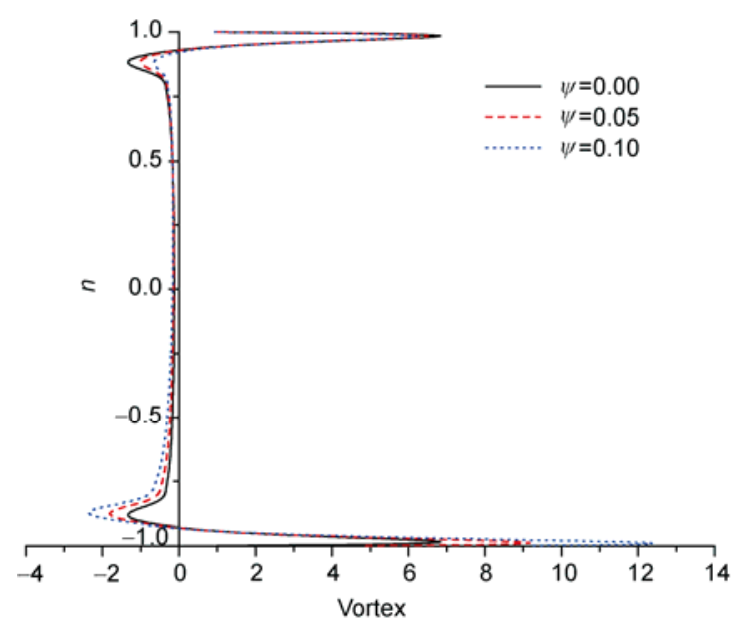

图 9 不同弯曲程度河湾中扰动浴量剖面

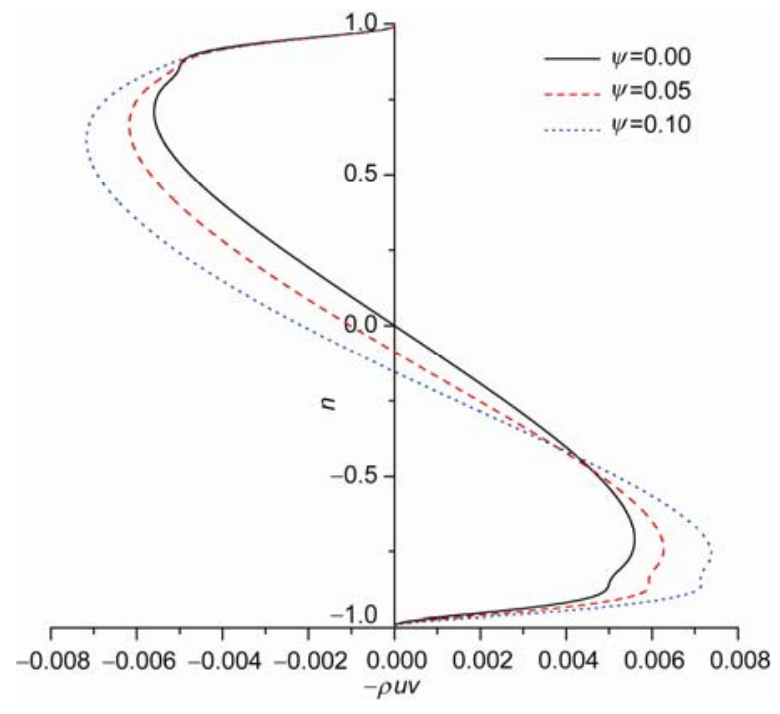

图 10 不同弯曲程度河湾中扰动切应力剖面

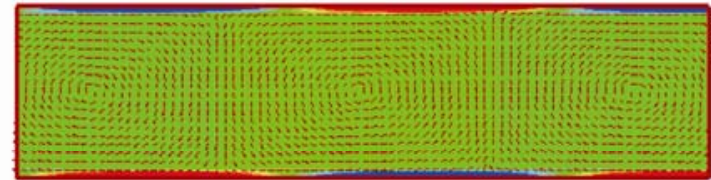

(a)

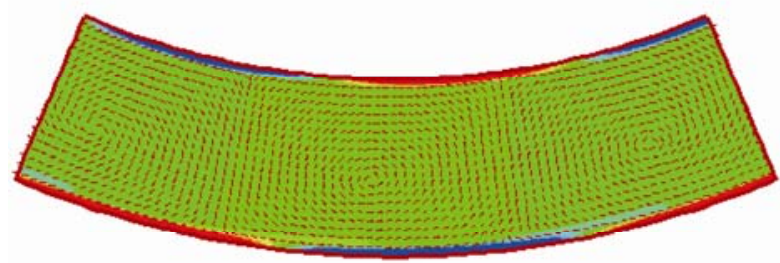

(b)

Vor: $-5-4-3-2-1 \quad 0 \quad 1 \quad 2 \quad 3 \quad 4 \quad 5$

图 11 顺直河道与弯曲河道中的扰动流场

(a) 顺直河道; (b) 弯曲河道

且各模态在横断面上亦表现出互异的特征. 紊流中 占主导地位的模态 1 , 其纵向拟序流速分布、切应力 分布向两岸近壁处集中，并呈反向分布，形成漩浴 (见图 11); 模态 2 和模态 3 的纵向拟序扰动流速、切 应力则向靠近中心线集中, 且正负变化剧烈, 形成尺 度不同的漩浴; 其他后序模态(如模态 4 等), 其峰值 区域也有各自的位置选择, 呈现不同的结构特征. 具 体如下.

模态 1 的纵向扰动流速沿横剖面由正到负, 产生 了图 11 所示的扰动流漩涡; 模态 2 和模态 3 的流速 剖面正负变化更为剧烈, 对应的扰动流也更加复杂, 正是由于这些不同模态各自所对应的扰动流场的复 杂性, 使得紊流中出现各种不同尺度的涡.

但值得注意的是, 本文所得流速数据均为 $\mathrm{O}-\mathrm{S}$
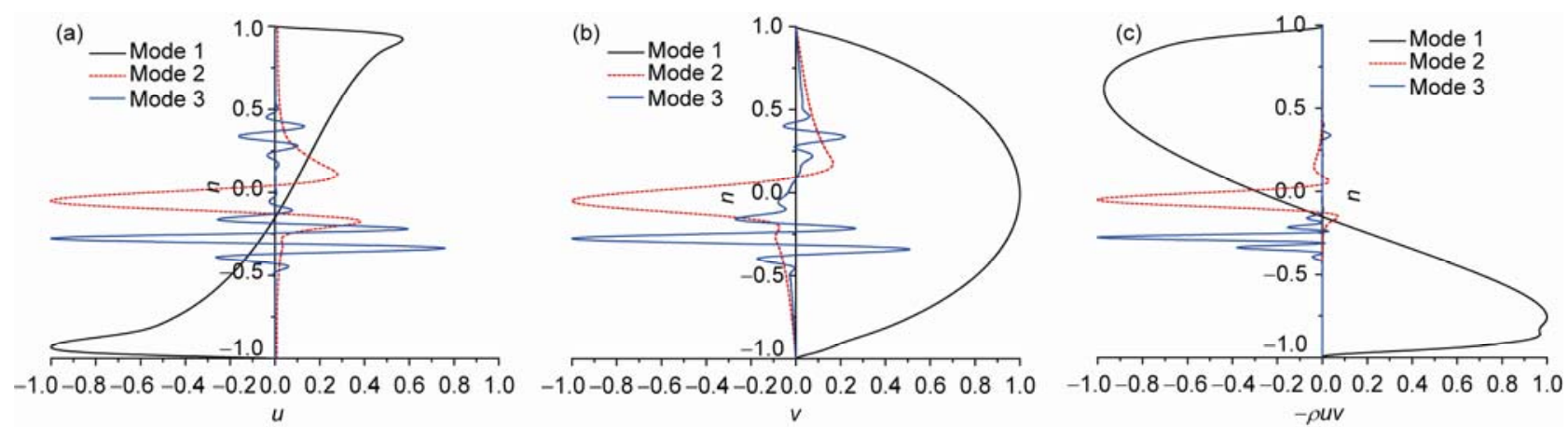

图 12 模态 1 3 的扰动量剖面

(a) 纵向扰动流速 $(s$ 方向); (b) 横向扰动流速 $(n$ 方向); (c) 扰动切应力 
方程的线性解, 仅表示紊流拟序扰动量在横向分布 的相对大小. 实际上, 当扰动流速大到一定程度时, 扰动控制方程中的非线性项作用将不能忽略, 这时 需要进行非线性特征分析.

\section{4 结论}

本文从理论上讨论了窄深型常曲率河湾紊流涡 体结构的特征. 采用曲线坐标以适合弯曲河流特征 分析, 运用差分法离散方程, 利用 Muller 法进行特征 值求解. 通过理论及数值计算, 得到了河湾紊流特 性.

1) 河湾对河道中的特征波有一定的选择性. 与
顺直河道相比, 河道弯曲使不稳定波段缩窄, 对紊流 拟序结构选择性增强, 也正是这些特征波段的紊流 结构, 促使了河湾的形成与保持.

2) 不同弯曲程度的河湾中各模态的特征波波速 和波速增长率表现出不同的变化特征. 随着弯曲程 度的增大, 河道中的特征波更加稳定, 这种趋势使得 弯曲河道的临界雷诺数比顺直河道大, 流态更稳定, 这也是自然界中, 顺直河流不易保持, 而弯曲河流和 河湾常常得以出现并长期保持的另一原因.

3) 弯道内的紊流结构有别于顺直河道, 其基本 流、扰动流剖面均向凹岸偏斜, 使得凹岸切应力大于 凸岸. 弯曲河道比顺直河道更易发生岸壁冲蚀, 凹岸 冲蚀、凸岸淤积, 使得微弯河流逐步向有限弯曲演变.

\section{参考文献}

1 张俊勇, 陈立, 刘林, 等. 汉江中下游河道最佳弯道形态. 武汉大学学报(工学版), 2007, 1: 37-41

2 万强, 江恩惠, 张林忠. 河湾平面形态及河湾流路方程研究综述. 人民黄河, 2009, 12: 127-129

3 覃莲超, 余明辉, 谈广鸣, 等. 河湾水流动力轴线变化与切滩拌弯关系研究. 水动力学研究与进展 A 辑, 2009, 1: 29-35

4 谈广鸣, 卢金友. 河道主流摆动与切滩演变初步研究. 武汉水利电力学院学报, 1992, 2: 107-112

5 Ishigaki T, Shiono K, Rameshwaran P. PIV and LDA measurements of secpndary flow in a meandering channel for overbank flow. J Vis, 2002, 5: 153-159

6 Kanhu C P, Srijib K Kar, Amartya K B. Flow and velocity distribution in meandering compound channels. J Hydrol Eng, 2004, 130: 398-411

7 Du H Q, Miwa H, Experimental study on the relation of bed morphology with surface flow in meander channels. J Sediment Res, 2006, 21: $1-12$

8 Yang F G, Liu X N, Cao S Y et al. Study on bed load transport for uniform sediment in laminar flow. Sci China Tech Sci, 2010, 53: 2414-2422

9 Wilson C A M E, Boxall J B, Guymer I, et al. Validation of a three-dimensional numerical code in the simulation of pseudo-natural meandering flows. J Hydrol Eng, 2003, 129: 758-768

10 Nils Reidar B. Olsen., Three-Dimensional CFD modeling of self-forming meandering channel. J Hydrol Eng, 2003, 129: 366-372

11 吴修广, 沈永明, 潘存鸿. 天然弯曲河流的三维数值模拟. 力学学报, 2005, 37: 689-696

12 王海娇, 王大国, 熊巨华, 等. 基于特征线的 N-S 方程算子分裂有限元法. 中国科学：技术科学, 2011, 41: 1143-1152

13 丁道扬, 吴时强. 用 $\kappa-\varepsilon$ 紊流模型计算跌坎水流数值方法探讨. 中国科学: 技术科学, 2011, 41: 441-448

14 白玉川, 徐海珏. 高含沙水流流动稳定性特征的研究. 中国科学: 技术科学, 2008, 38: 135-155

15 Bai Y C, and Andreas M, A linear disturbance theory for coherent structure and mechanic sediment research. J Sediment Res, 2001, 16: 234-243

16 白玉川, 罗纪生. 明渠层流失稳与沙纹成因机理研究. 应用数学与力学, 2002, 23: 254-268

17 白玉川, 徐海珏. 沙纹床面明渠层流稳定性特征的研究. 中国科学 E 辑: 技术科学, 2005, 35: 53-73

18 Yalin M S. Mechanics of Sediment Transport. 2nd Edition. Pergamon Press, 1977, 259

19 Bai Y C, Han Q W, Xu H J, et al. Theory and applications of nonlinear river dynamics. In: Proceeding of the 7th IAHR symposium on River, Coastal and Estuarine Morphodynamics, Beijing, 2011

20 周恒, 陆昌根, 罗纪生. 紊流边界层近壁区单个相干结构的模拟. 中国科学 A 辑: 数学, 1999, 29: 366-372

21 罗纪生, 周恒. 紊流边界层外区大尺度相干结构的理论模型及实验的比较. 中国科学 A 辑: 数学, 1993, 23: 56-62

22 Zhang Z, Lilley G M. A theoretical model of the coherent structure of the turbulent boundary layer in zero pressure gradient. In: Turbulent shear flows 3, International Symposium, 3rd, Davis, CA, September 9-11, 1981, Selected Papers (A83-33651 15-34). Berlin: Springer-Verlag, 1982.60-72

23 张东明, 罗纪生, 周恒. 紊流边界层近壁区相干结构的动力学模型. 中国科学 $\mathrm{G}$ 缉: 物理 力学 天文学, 2003, 33: 76-82

24 白玉川, 杨燕华. 弯曲河流水流动力稳定性特征研究. 中国科学: 技术科学, 2011, 41: 971-980 